Meta

Journal des traducteurs

Translators' Journal

\title{
La traduction et ses discours
}

\section{Antoine Berman}

Volume 34, numéro 4, décembre 1989

URI : https://id.erudit.org/iderudit/002062ar

DOI : https://doi.org/10.7202/002062ar

Aller au sommaire du numéro

Éditeur(s)

Les Presses de l'Université de Montréal

ISSN

0026-0452 (imprimé)

1492-1421 (numérique)

Découvrir la revue

Citer cet article

Berman, A. (1989). La traduction et ses discours. Meta, 34(4), 672-679.

https://doi.org/10.7202/002062ar

Ce document est protégé par la loi sur le droit d'auteur. L'utilisation des services d'Érudit (y compris la reproduction) est assujettie à sa politique d'utilisation que vous pouvez consulter en ligne.

https://apropos.erudit.org/fr/usagers/politique-dutilisation/ 


\title{
LA TRADUCTION ET SES DISCOURS
}

\author{
ANTOINE BERMAN \\ Centre Jacques Amyot, Paris, France
}

Je me propose d'examiner ici brièvement quels sont les différents «discours» tenus sur la traduction. Il s'agira d'analyser ceux qui existent déjà, et d'en proposer un autre. La prétention de ce propos sera atténuée, je l'espère, par le fait que ce «nouveau» discours s'enracine dans la traditionnalité la plus traditionnelle. Je mènerai cette réflexion à partir d'un triple horizon personnel: en tant que traducteur de plusieurs domaines et de plusieurs langues, en tant que «théoricien» de la traduction donnant des séminaires au Collège international de philosophie, et en tant que membre d'un organisme gouvernemental français, le Commissariat général de la langue française, qui développe actuellement une politique de la traduction.

\section{LE DISCOURS «TRADITIONNEL» SUR LA TRADUCTION}

Les traducteurs n'aiment généralement guère parler «théorie». Ils se conçoivent comme des intuitifs et des artisans. Et pourtant, dès le début de la tradition occidentale, l'activité traduisante s'est accompagnée d'un discours-sur-la-traduction. Ainsi avonsnous au fil des siècles (pour ne citer que les noms les plus connus), les textes de Cicéron, Saint-Jérôme, Fray Luis de Léon, Luther, Du Bellay, Dolet, Rivarol, Herder, Humboldt, A.W. Schlegel, Goethe, Schleiermacher, Chateaubriand, Pouchkine, Valéry, Benjamin, Pound, Armand Robin, Borges, Bonnefoy, Octavio Paz, etc. Ce discours est pour l'essentiel celui des traduisants, même s'il se double, à chaque époque, de celui des non-traduisants, qui ne fait que le refléter et le répéter. Je l'appelle le discours «traditionnel». Traditionnel, il l'est en deux sens. Premièrement, il nous vient du fond de la tradition culturelle occidentale. Deuxièmement, il appartient à un monde où la traduction est considérée comme l'un des piliers de la traditionnalité, c'est-à-dire du mode d'être des hommes, déterminé par quelque chose comme la tradition. Traduzione tradizione, disent les Italiens ; unissant passé et présent, proche et lointain, la traduction ensemence la culture, elle-même expérimentée comme un ensemble de traditions.

Ce discours a trois caractéristiques. Il est d'abord disparate: tantôt analytique et descriptif, tantôt prescriptif, tantôt lyrique, tantôt spéculatif, tantôt polémique, il est rarement «théorique» au sens moderne. De fait, le premier texte «théorique» sur la traduction est probablement celui de Schleiermacher, Des différentes méthodes de traduire (Berlin, 1821) $)^{1}$.

Ce discours, ensuite, est d'une étonnante minceur: peu d'ouvrages, une masse de notes, de lettres, de préfaces, etc.; et si l'on compare ce corpus à celui des textes «critiques» que la littérature a produits sur elle-même depuis, en gros, la Renaissance, on devra en conclure que les traducteurs sont fort parcimonieux lorsqu'ils parlent de leur activité. Tout se passe comme si la traduction n'osait pas s'affirmer sur un mode discursif. Néanmoins, malgré la minceur, ce discours est riche, très riche, et nous devons apprendre à le lire. À le connaître, car nous le connaissons encore très mal.

La troisième caractéristique est la suivante: ce discours traditionnel est marqué par une dissension, celle des partisans de la «lettre» et des partisans du «sens», ces derniers étant toujours majoritaires. Cette dissension (nous y reviendrons) a pour fondement la 
double potentialité du traduire, et non des «préférences» sociales ou subjectives que l'on pourrait avoir à ce sujet.

Face à ce discours traditionnel, le $\mathrm{XX}^{\mathrm{e}}$ siècle a vu se constituer une multiplicité de discours nouveaux sur la traduction, qui sont tantôt des discours «objectifs», tantôt des discours d' «expérience». Voyons d'abord les discours «objectifs». Ils sont tantôt sectoriels (liés à des disciplines définies), tantôt généraux (théories générales de la traduction).

\section{LES DISCOURS OBJECTIFS SECTORIELS}

Ce sont essentiellement ceux de la linguistique, de la poétique (ou sémiotique) et de la littérature comparée. Je laisse de côté ici les discours sur la traduction juridique, technique et orale (interprétation), pragmatiques et, à peu d'exception près, encore faiblement systématisés 2 .

Les analyses que la linguistique a consacrées à la traduction sont relativement peu nombreuses. Les plus notables sont celles de Jakobson, de Catford et de Nida. En théorie, la linguistique affirme que la traduction est pour elle un thème essentiel, une opération dont elle doit montrer la possibilité ou, éventuellement, l'impossibilité. Il s'agit pour elle, a priori, d'un phénomène d'interaction entre deux langues, qu'elle définit en formalisant le concept courant de traduction. Ainsi aboutit-on à des formules comme, chez Jakobson, la «recherche de l'équivalence dans la différence». La linguistique donne ainsi une définition tellement vaste et tellement abstraite du traduire qu'elle omet presque complètement sa dimension écrite et textuelle, pour ne pas parler de ses dimensions culturelles, historiques, etc. Tout cela paraît renvoyer à un certain désintérêt pour un «objet» qu'elle persiste à inclure dans son domaine de compétence, même si - par hasard - la linguistique a fourni le cadre catégoriel des analyses sémiotiques et stylistiques de la traduction. Pourquoi ce désintérêt? Il conviendrait de s'interroger sur ses causes.

La poétique considère la traduction comme une forme d'hypertexte ou de métatexte. Si la linguistique néglige la dimension textuelle du traduire, elle néglige, quant à elle, sa dimension langagière. Là encore, on constate un certain désintérêt, comme il est patent dans Palimpsestes de Gérard Genette, dont les analyses de la parodie, du pastiche, de l'imitation, sont bien plus poussées que celles de la traduction. Ainsi la poétique commence-t-elle seulement (dans la foulée de Lotman) l'étude des structures de traduisibilité et de traductivité des œuvres littéraires, sans parler de celle de la structure textuelle des traductions elles-mêmes.

La littérature comparée, étudiant les interactions des systèmes littéraires, ne pouvait à la longue négliger la traduction. Avec un assez grand retard, elle produit maintenant des analyses de la place de celles-ci dans les corpus littéraires. C'est même là que nous trouvons, au niveau du savoir institué, un intérêt accru pour la traduction, celui qui parât manquer chez les linguistes et les «poéticiens». Toutefois, la traduction n'est pour elle que l'un des modes d'interaction des textes, et elle ne peut aborder l'aire de la traduction en tant que telle, qui déborde forcément le «littéraire», fût-il défini au sens le plus large.

\section{LES DISCOURS GÉNÉRAUX}

Il s'agit de ce qu'on appelle de nos jours les «théories» de la traduction. Ces dernières ont une double base : $l^{\prime}$ herméneutique de la compréhension du XIX $\mathrm{X}^{\mathrm{e}}$ siècle (c'est le cas de Steiner) et la linguistique (c'est le cas de Nida, Mounin, les Russes). Cela signifie d'abord que ces théories ne sont jamais autonomes; qu'elles ne sont qu'une sous-partie d'un ensemble plus vaste. Ainsi Vinay et Darbelnet rangent-ils l'étude de la traduction dans la «linguistique appliquée». Ces théories, ensuite, partent elles aussi d'une définition a priorique de la traduction comme «processus de communication interlinguistique»; à 
partir de là, elles s'efforcent de construire des typologies et débouchent avec une belle régularité sur des propositions d'ordre prescriptif et méthodologique. Bien des fois, comme les discours sectoriels, elles émanent de spécialistes qui ne sont pas des traducteurs : d'où le fameux hiatus entre les «théoriciens» et les «praticiens», les seconds méprisant les «constructions abstraites» des premiers, et ces derniers méprisant l'empiricité muette des seconds. Mais là n'est pas l'essentiel. Car ces discours se fondent sur la présupposition que l'on peut édifier une théorie globale et unique du traduire, qu'il s'agisse de poésie, de théâtre, de prose littéraire, de philosophie, de textes techniques ou juridiques, de langues proches ou lointaines, vivantes ou mortes, orales ou écrites, communes ou dialectales, de premières traductions ou de retraductions, d'hétéro-traductions ou d'auto-traductions, etc. Elles négligent le fait que l'espace de la traduction est irrémédiablement pluriel, hétérogène et non unifiable. Certes, elles récusent à bon droit l'empirisme naïf des traducteurs, pour lesquels aucun discours général ne peut être tenu sur leur activité. Mais cela signifie-t-il que l'on puisse faire tenir dans un concept unique - sous prétexte de «scientificité» - tous les modes de traduction? Et si l'on parvient à le faire, sur quelle base? À quel prix ?

Il est vrai qu'un autre corpus théorique sur la traduction se développe depuis quelques années: celui représenté par ce qu'on appelle l'«école de Tel Aviv» (EvenZohar, Gidéon Toury) et tous ceux qui, un peu partout, suivent ses axes programmatiques (comme José Lambert à Louvain). Aux théories classiques, dogmatiques et prescriptives, l'«école de Tel Aviv» oppose une théorie de la «littérature traduite» et de la place de celle-ci dans les "polysystèmes» littéraires. Even Zohar et Toury refusent de partir d'un concept a priorique du traduire : ils s'attachent à étudier ce qui, dans tel ou tel système littéraire (et culturel) est posé comme «traduction». Ainsi veulent-ils éviter l'écueil de la normativité et constituer une science du traduit, faisant elle-même partie d'une science de tous les «transferts» interculturels. On peut néanmoins se demander si ce savoir purement descriptif de la traduction est en soi suffisant. Car un tel savoir, tout en échappant à l'abstraction des théories classiques, met entre parenthèses la question de la vérité de la traduction. Lorsque nous disons, par exemple, que les «vraies» traductions sont rares, nous ne partons pas d'un concept dogmatique du traduire, mais d'une expérience de celui-ci où il s'agit de la vérité du rapport aux ouvres. Le descriptivisme de l'«école de Tel Aviv» qui permet la constitution d'un riche corpus sur toute la masse du «traduit» et ses déterminations socioculturelles - rencontre là sa limite. Ses présupposés (comme ceux des théories antérieures) doivent donc être soumis à une critique systématique. Peut-être (on y reviendra) l'idée même d'une «théorie» de la traduction, fut-elle purement descriptive $^{3}$, est-elle un leurre. Cela, bien sûr, si nous prenons le concept de «théorie» au sens strict, tel qu'il se présente dans le domaine des sciences. Tout discours articulé n'est pas théorie.

\section{LES DISCOURS D'EXPÉRIENCE}

Deux autres discours, au XXe siècle, prennent en vue, passionnément, la traduction. Sur un mode qui n'est pas théorique, mais néanmoins conceptuel. Le premier est celui de la philosophie. Par une nécessité qui tient au destin moderne de ses interrogations, la philosophie est concernée au plus près par la traduction, comme on voit avec Benjamin, Heidegger, Cadamer, Derrida, Serres et, dans le domaine de la pensée dite «analytique», Wittgenstein et Quine. Il ne s'agit pourtant pas de «philosophie de la traduction», mais, ce qui est plus déroutant, d'un entrelacement du philosopher et du traduire. Ainsi la pensée de Heidegger est-elle pour une bonne part un travail-de-traduction.

Le second est celui de la psychanalyse. Celle-ci est doublement concernée par la traduction. D'abord, parce qu'elle est liée à un texte fondateur, celui de Freud, dont le 
«destin de traduction» fait problème. Ensuite, parce que Freud lui-même a parfois défini en termes de traduction, d'Übertragung, de transfert, qui signifie aussi «traduction» en allemand. Il n'y a ni «psychanalyse de la traduction», ni «théorie psychanalytique» de celle-ci, mais un corpus croissant de réflexions s'efforçant d'approfondir le lien d'essence de la psychanalyse au traduire, dans le cadre de toute une méditation sur le sujet, l'inconscient, la langue et la lettre. Ce corpus ne peut être ignoré, même si son développement ne peut être l'œuvre que des seuls psychanalystes.

\section{LA TRADUCTIQUE}

Un dernier discours, enfin, qui s'ignore encore comme tel, se profile aujourd'hui à l'horizon. Il est technologique, et se constitue actuellement au carrefour de la théorie de l'information, de la théorie de l'intelligence artificielle, de la terminologie, de la linguistique et de l'informatique. Je l'appelle la traductique. Pour ce discours, la totalité des processus pris en vue par la science et la technique constitue un vaste système de commutations, de permutations et de computations qu'il semble pertinent d'analyser en termes de traduction, au sens du «change» généralisé et formalisé de tout en tout, de «l'omni-translation où, idéalement, tout circule ${ }^{4}$ ». La traductique est (sera) la théorie computationnelle des processus traductifs régissant l'aire technologique, ou le réel technologiquement appréhendé. On en trouve les linéaments, entre autres, dans les recherches touchant à la traduction assistée par ordinateur et à l'analyse informatico-linguistique des langues naturelles.

Si elle concerne a priori l'aire technologique, il est clair que la traductique déborde déjà largement celle-ci. Par exemple, le système de traduction assistée par ordinateur Weidner a dès le début été conçu pour traduire... la Bible. Le but de la traductique est évidemment de produire un discours théorico-pragmatique touchant tous les domaines de traduction, y compris «littéraires». Le jour est proche où elle s'annexera le structuralisme et le fonctionnalisme de la sémiotique à cette fin. D'ores et déjà, l'informatique bouleverse toute la pratique de la traduction, à un degré que nous mesurons encore mal.

Or, curieusement, cet élément computationnel du traduire auquel s'attache la traduction correspond à une dimension computationnelle du «littéraire» lui-même. D'abord, parce qu'un texte est un système qui peut, et doit, faire l'objet de procédures de traduction elles-mêmes systématiques. Mais il y a plus: de Novalis et Hölderlin à Poe, Valéry, Musil et les poètes et formalistes russes, la littérature s'est elle-même conçue comme un «calcul». Si bien que ce qu'énonce la traductique est secrètement lié à un certain destin moderne du «littéraire».

Toutefois, ce nouveau discours, dans la mesure même où il se veut «scientifique», est privé de réflexivité propre: on ne peut pas penser en termes de technologie sur celleci. Pour le faire, il faut sortir du «langage» de la technologie. Cela, la traductique ne peut le faire.

\section{LA TRADUCTOLOGIE}

Il appartient à un discours tout différent de déployer, pour le traduire, l'élément de la réflexivité. Je propose de lui réserver le terme de traductologie, même si certains l'emploient déjà pour désigner un savoir objectif de la traduction.

La traductologie est la réflexion de la traduction sur elle-même à partir de sa nature d'expérience. Essayons de préciser cette définition. Réflexion et expérience: voilà des catégories que la philosophie n'a cessé de méditer, avec Kant, Fichte, Hegel, Husserl, Benjamin et Heidegger. Dont elle n'a cessé de méditer l'unité. Car lorsque l'expérience se retourne sur elle-même pour se saisir et devenir plus pleinement «expérience», elle devient réflexion. Plus exactement, la réflexion n'est rien d'autre qu'un tel retournement, 
qui s'effectue dans le medium de la langue naturelle. Telle est la structure «spéculative» qu'interroge la philosophie. Mais pas seulement elle: depuis les Romantiques, la littérature aussi. Ainsi de Proust, déclarant à propos de l'écriture comme mémoire qu'en elle «le pouvoir réfléchissant est tout». Certes, ce romancier semble partir d'expériences singulières mais, par la réflexion de l'écriture, ces expériences s'universalisent. Proust déclare à ce propos que l'écrivain «ne se souvient que du général 15 ». Et cela, dans le même passage du Temps retrouvé où il définit l'acte d'écrire comme une traduction. Pour lui, réflexivité et traductivité de l'œuvre sont liées ${ }^{6}$.

La traductologie est donc la reprise réflexive de l'expérience qu'est la traduction, et non une théorie qui viendrait décrire, analyser et éventuellement régir celle-ci.

L'expérience faite dans la traduction a une triple dimension.

En premier lieu, le traducteur fait l'expérience de la différence et de la parenté des langues, à un niveau qui dépasse ce que la linguistique ou la philologie peuvent empiriquement constater à ce sujet, puisque cette parenté et cette différence se manifestent dans l'acte de traduire lui-même.

En second lieu, il fait l'expérience de la traduisibilité et de l'intraduisibilité des ceuvres.

En troisième lieu, il fait l'expérience de la traduction elle-même en tant qu'elle est marquée par deux possibilités antagonistes, être restitution du sens ou réinscription de la lettre. On voit que dans chaque dimension, il y a une structure de dissension. C'est elle qui est à l'origine des sempiternelles controverses sur le caractère «problématique» du traduire. La traductologie entend reprendre dans une réflexion systématique ces trois dimensions de l'expérience traductive. Elle continue ainsi le discours traditionnel là où il s'arrête, c'est-à-dire au seuil de la systématicité.

Ce n'est plus un discours sur la traduction, mais un discours enraciné dans cette expérience triplement dissensive. Il n'est ni «scientifique», ni «littéraire». Il ne remplace pas (et n'ambitionne pas de remplacer) la linguistique, la sémiotique, la littérature comparée, etc. Il se tient bien plutôt à côté de ces savoirs. Il est l'équivalent, pour la traduction, du discours critique de la littérature sur elle-même. Musil disait que la critique était «tissée» à la littérature. Le discours traductologique, lui, se fonde sur la réflexivité originaire du traduire.

L'aire des traductions n'étant pas close, mais éclatée et interstitielle, la traductologie n'est pas un discours fermé qui prendrait en vue tel champ du réel: justement, l'aire de la traduction n'est pas un «champ», au sens que prend ce concept dans les sciences.

Par contre, la traductologie récuse d'entrée de jeu l'idée d'une théorie globale et unique du traduire. Une telle théorie n'est possible que dans l'horizon de la restitution du sens. Or, celle-ci est une dimension réelle, mais seconde, des traductions. C'est bien leur seul point commun à toutes, mais le plus problématique, car il occulte une autre dimension plus essentielle: le travail sur la lettre. C'est en tant que travail sur la lettre que la traduction joue un rôle éthique, poétique, culturel et même religieux dans l'histoire.

\section{LES TÂCHES D'UNE TRADUCTOLOGIE}

Voyons maintenant quelles sont les tâches possibles d'une traductologie.

La première est négative. Étant donné - comme le dit Steiner - que $80 \%$ des traductions sont «fautives», il convient d'analyser les facteurs déformants opérant dans le traduire et l'empêchant d'atteindre sa pure visée. Telle est la tâche d'une analytique de la défaillance et d'une analytique de la destruction.

L'analytique de la défaillance prend en vue ce que Freud appelle (pour le psychisme) «le défaut de traduction», c'est-à-dire le fait que l'acte de traduire ne se réalise jamais (pleinement) même quand cela est possible. Le «défaut de traduction» revêt de multiples formes, mais il est inhérent à toute traduction. 
L'analytique de la destruction prend en vue le fait que le traduire, en tant que restitution du sens (et il l'est toujours) est un processus de dégradation de la lettre des ouvres. Là encore, ce processus se manifeste comme une série de tendances déformantes opérant au cours de la traduction (que le traducteur le veuille ou non, qu'il obéisse ou non à des normes culturelles, littéraires, morales, etc.). Cette destruction n'est pas que négative. Elle a même sa nécessité. Car l'un des rapports possibles de l'homme à ses œuvres est précisément la destruction. Glose et traduction, comme le sentait bien Montaigne, sont iconoclastes.

La seconde tâche de la traductologie consiste à expliciter ce qui, dans la traduction, relève d'autre chose que de la communication des contenus et de la restitution du sens: le travail sur la lettre. C'est le domaine d'une éthique et d'une poétique de la traduction, dans la mesure où l'éthique et la poésie n'existent que dans le «respect» (l'observance) de la lettre.

La troisième tâche se rapporte à la temporalité et à l' historicité des actes de traduction. Les traductions ont une temporalité propre, qui est liée à celle des œuvres, des langues et des cultures. Cette réflexion sur le temps du traduire ouvre à une étude de caractère «historique»: écrire l'histoire de la traduction dans les aires où elle a constitué l'un des facteurs fondamentaux (encore méconnu comme tel) de la constitution des langues et des littératures. Ce travail historique, semblable à celui de Michel Foucault, fera apparaître que partout et toujours, traduction et écriture forment une unité originaire. Le paradoxe central d'une histoire de la traduction étant peut-être que l'histoire ellemême commence avec la traduction.

La quatrième tâche consiste à analyser l'espace pluriel des traductions, sans confondre ce travail avec la constitution d'une «typologie», aussi affinée qu'elle puisse être. Cet espace peut être approché selon des axes totalement hétérogènes. La traduction d'un livre d'enfant n'obéit pas aux mêmes «lois» que celle d'un livre pour adultes; celle d'un texte technique differe de celle d'un texte scientifique, juridique, publicitaire, commercial et, naturellement, «littéraire», l'espace du «littéraire» étant à son tour fondamentalement hétérogène et - en particulier - scindé entre ce qui est «œuvre» et ce qui, quoique «littéraire» n'est pas œuvre. Ainsi, un texte écrit en dialecte ne se traduit pas comme un texte écrit en koiné; un texte écrit en français par un étranger ne se traduit pas comme un texte écrit en français par un français; une première traduction ne peut être lue comme une «re-traduction», une auto-traduction comme une «hétéro-traduction», une traduction de langue «lointaine» comme une traduction de langue «proche», etc. Tout cela n'est pas unifiable.

La cinquième tâche de la traductologie consiste à développer une réflexion sur le traducteur, car on peut bien dire que celui-ci est le grand oublié de tous les discours sur la traduction. Pour ceux-ci, le traducteur est un être sans épaisseur, «transparent», «effacé», etc. C'est du reste de la sorte que s'imaginent et se vivent les traducteurs eux-mêmes, qu'ils soient «techniques» ou «littéraires». Or, il n'en est pas ainsi. On peut imaginer ici des «biographies» de traducteurs comme Amyot, A.W. Schlegel, Armand Robin, des analyses de destins-de-traduction où s'éclairerait le rapport du traducteur à l'écriture, à la langue maternelle et aux autres langues. Cette analytique du traducteur, à ma connaissance, n'existe pratiquement pas. Dans le même ordre d'idée, il serait possible d'étudier comment apparaissent dans la littérature le traducteur et la traduction; de fait, ils y apparaissent peu, mais cette apparition, à chaque fois, est fort significative.

La sixième tâche consiste à analyser pourquoi, et de tout temps, la traduction a été une activité occultée, marginalisée, dévalorisée, qu'elle soit travail sur la lettre ou libre restitution du sens. 
La septième tâche consiste à explorer, si l'on peut dire, les bords de la traduction. Et cela selon deux axes.

Sur ses bords «horizontaux», l'aire du traduire touche d'autres aires: celle de la lecture, celle des «interprétations», celles des transferts et changes en tous genres, qu'ils soient littéraires, artistiques, scientifiques, etc. Grande est la tentation, ici, d'édifier une théorie de la «traduction généralisée» qui engloberait la «traduction restreinte» et les autres modes de «translation». À cette tentation ont succombé le Romantisme allemand, Steiner, Serres et, en France, la revue «Change». La tâche de la traductologie consiste bien plutôt à articuler, sans les confondre, toutes ces aires de transformation.

Sur ses bords "verticaux», la traduction connaît un changement de sens métaphorique, quand elle en vient à désigner l'essence des actes de parole, d'écriture, de pensée et même d'existence. Cet emploi métaphorisant du «concept» de traduction est déjà constant dans le discours quotidien, mais il a été radicalisé par une longue lignée d'auteurs, au moins depuis le XVIII' siècle.

Hamann

Parler, c'est traduire - d'une langue angélique en une langue humaine?

Marina Tsvetaïeva:

Traduire (...) c'est refrayer la voie sur des traces que l'herbe envahit dans l'instant, mais (...) aussi autre chose. On ne fait pas seulement passer une langue dans une autre langue (le russe par exemple), on passe aussi la rivière. Je fais passer Rilke en langue russe, tout comme il me fera passer un jour dans l'autre monde ${ }^{8}$.

Proust

Il est probable que si une traduction complète de l'univers pouvait être donnée, nous serions devenus éternels9.

\section{Ros Bastos}

Il n'y a qu'un seul volume. Quand un homme meurt, cela ne signifie pas que ce chapitre soit arraché aux pages du Livre. Cela veut dire qu'il a été traduit dans une langue meilleure. Chaque chapitre est ainsi traduit ${ }^{10}$.

Il y a là une métaphorique verticale de la traduction qui ne saurait être ignorée : on peut parler de l'autre traduction qui se cache dans la traduction restreinte comme son noyau le plus secret.

La huitième tâche de la traductologie consiste à faire une «critique de la raison traductique», c'est-à-dire à définir les limites de validité de celle-ci. Prise dans le mouvement impérieux de la technologisation de la langue, la traductique ne peut se fixer à elle-même ses indispensables bornes épistémologiques, culturelles et même politiques. Cela est d'autant plus nécessaire qu'aujourd'hui, la traduction est entrée pleinement dans l'espace des «politiques» (et du politique comme tel).

La neuvième tâche consiste à définir les rapports de la traductologie comme discours-de-la-traduction avec deux autres modes essentiels de rapport aux œuvres: le commentaire et la critique. Cette tâche est d'autant plus importante que la traduction a souvent été définie comme une activité critique (c'est le criticism by translation de Pound) ou été inféodée à l'activité critique (du Romantisme allemand à Steiner); et que, par ailleurs, commentaire et traduction entretiennent des rapports intimes, comme le montrent, au $\mathrm{XX}^{\mathrm{e}}$ siècle, les réflexions philosophiques, religieuses et psychanalytiques. 
La dixième tâche de la traductologie consiste à définir les conditions de sa propre institutionnalisation en tant que savoir autonome. Il s'agit de préciser les conditions d'un enseignement et d'une recherche. Si nous considérons que la traduction est pour nous essentielle, nous concerne tous, que le destin de l'homme, «Babel», est et restera un destin-de-traduction, alors quelque chose comme une traductologie doit exister comme savoir institué, même si ce savoir ne débouche pas sur une science, une Übersetzungwissenschaft. Fait partie de cette institutionnalisation (dont les modalités concrètes doivent être précisées) ce qu'on pourrait appeler l'éducation-à-la-traduction. La mise en place d'une telle éducation, d'une telle paideia traductive ${ }^{11}$ devrait à son tour modifier le statut de la traduction dans notre culture, la figure du traducteur et, naturellement, en aval, tout ce qui se veut aujourd'hui enseignement pratique de la traduction.

La onzième tâche de la traductologie se rapporte au lien que toute réflexion sur la traduction entretient avec la tradition-de-la-traduction particulière à laquelle elle appartient, même si son ambition est de constituer un discours «universel». La manière dont apparaît la problématique de la traduction n'est pas la même dans la tradition française que dans la tradition allemande, anglo-saxonne, russe, espagnole ou - a fortiori extrême-orientale. Elle n'est pas la même dans un «petit pays» dont la langue est uniquement nationale que dans un grand pays dont la langue est transnationale et dont l'espace est lui-même souvent polylingue, etc.

Il incombe donc à la traductologie de se saisir comme un discours historiquement et culturellement situé, et d'étudier, à partir de cette situation — de $s a$ situation - les autres discours sur la traduction. Ainsi, derrière les théories de Nida se profile une problématique de la traduction propre à l'espace anglo-saxon; derrière tel écrit d'Efim Etkind, une problématique propre à l'espace russe; derrière les réflexions de Yebra, une problématique propre à l'espace hispanique; derrière les constructions théoriques et les pratiques d'Octavio Paz ou d'Haroldo de Campos, une problématique latino-américaine de la traduction, etc. La traductologie est donc toujours liée à l'espace de langue et de culture auquel elle appartient, et il est bien évident que les grands axes de réflexion que nous avons proposés ici s'enracinent, même si c'est pour la contester, dans la tradition française de la traduction. Cela n'enlève rien à son universalité; mais cela ouvre à la nécessité d'un dialogue entre les différentes traditions de réflexion sur la traduction. Il en va de même, au fond, pour la littérature, la pensée, le théâtre ou la psychanalyse.

NOTES

1. Voir ma traduction de ce texte-clé dans les Tours de Babel, Toulouse, éd. Trans-Europ-Repress, 1985.

2. Mentionnons cependant, pour la traduction technique, les travaux de B. Folkart et, pour la traduction juridique, ceux de J.-C. Gémar (Ottawa et Montréal).

3. Resterait d'ailleurs à voir si la théorie descriptive n'opère pas un retour discret à la normativité. Cf. «Les théories de la traduction et le partage des champs discursifs : fonctionnalisme et caractérisation du littéraire», Annie Brisset, in Neonelicon, Budapest, 1986.

4. Cité dans les Immatériaux, Paris, Centre Pompidou, 1985.

5. Le Temps retrouvé, Pléiade, tome $\mathrm{III}$, p. 906.

6. Distinguons ici traduisibilité et traductivité. La traduisibilité est une structure a priori de l'œuvre qui la rend «traduisible», comme la criticabilité fait qu'on peut l'analyser critiquement. La traductivité renvoie au fait que le texte lui-même est un travail de traduction. Pasternak parle à cet égard de la «tension traductrice» de la grande prose.

7. Esthetica in nuce, Poésie, no 13 , Paris, 1980, p. 17 ; trad. J.-F. Courtine.

8. Correspondance à trois, Paris, Gallimard, 1983, p. 17 ; trad. P. Jaccottet.

9. La Matinée chez la Princesse de Guermantes, Paris, Gallimard, 1983, p. 580.

10. Moi le Suprême, Paris, Belfond, 1977 ; trad. A. Berman.

11. Que notre époque s'achemine vers une telle paideia, c'est ce qu'attestent les créations du Collège européen des traducteurs de Strahlen (Allemagne fédérale), du Collège international des traducteurs d'Arles et, un peu partout, de nombreux «centres de recherches» sur la traduction. La traductologie n'est rien d'autre que la réflexion qui vient soutenir et éclairer (tout en s'y développant et approfondissant) l'activité de ces centres et collèges. 